\title{
Solving the First Kind Fuzzy Integral Equations Using a Hybrid Regularization Method and Bernstein Polynomials
}

\author{
A. K. Moloodpour ${ }^{1} \&$ A. Jafarian ${ }^{1}$ \\ ${ }^{1}$ Department of Mathematics, Urmia Branch, Islamic Azad University, Urmia, Iran \\ Correspondence: A. Jafarian, Department of Mathematics, Urmia Branch, Islamic Azad University, Urmia, Iran. \\ E-mail: jafarian5594@yahoo.com
}

Received: April 12, 2016

doi:10.5539/mas.v10n9p22
Accepted: April 28, $2016 \quad$ Online Published: May 9, 2016

URL: http://dx.doi.org/10.5539/mas.v10n9p22

\begin{abstract}
A hybrid of regularization methodand Bernstein polynomials is used to solve the first kind fuzzy integral equations. In this paper, first the regularization method applied to convert the first kind fuzzy integral equation into the second kind fuzzy integral equation. Then by approximating Bernstein polynomials, the obtained second kind fuzzy integral equation is solved. In this method, one parameter was created in the second kind equation. When this parameter tends to zero, the solutions of integral equation of the second kind tend to solutions of the integral equation of the first kind. The obtained solutions are comparable to the solutions of the other similar methods. Performance of the mentioned method is illustrated by considering some example.
\end{abstract}

Keywords: fuzzy integral equation, Bernstein polynomials, regularization method

\section{Introduction}

Integral equations are employed in numerical Computations and in modeling of engineering science and other sciences (Jerri, 1999; Tikhonov, 1963; Wazwaz, 2011) as well. In manynonsingular integral equations of the first kind, especially Abel equations, the exact solution will not be achieved. In solving (Hanna, Roumeliotis \& Kucera, 2005) Fredholm integral equations of the first kind the local regularization method is employed. Integral equations of the first kind with single kernel were solved by applying the Cauchy equation in (Okecha \& Onwukwe, 2012). Chebyshev polynomials and Jacobi polynomials are considered in this paper. Chris et al. (Karimi \& Jozi 2015), have solved equations of the first kind using least squares. Another type of integral equations is called Fuzzy integral equations and is a very useful tool in several applications such as engineering areas. Fredholm fuzzy integral equations are solved by numerical method (Abbasbandy. et al (Abbas, Babolian \& Alavi, 2007)). Adomian decomposition method is employed to solve the Fredholm fuzzy integral equations of the second by Babolian, et al. (Babolian, Sadeghi \& Goghary, 2005). Another approach to solve the integral equations of the first kind is to transform them to the integral equations of second kind by regularization method. In (Wazwaz, 2011) the first kind integral equations have been solved by hybrid regularization and Homotopy method. Another type of the first kind equations is the Abel integral equation which is presented in Fuzzy form. Jafarian et al. (Jafarian \& Measoomy, 2014), solved Abel fuzzy integral equations by multi-layers neural network and "back-propagation learning". Here, first the regularization method was used for solving the non-fuzzy integral equation and integral equation of the first kind. Then considering transformation of the first kind equations to the second kind equations by regularization method, we need to solve the obtained second kind equation by choosing Bernstein polynomials. Then integral equation of the second kind will be solved by Bernstein polynomials Approximation. Finally, by hybridregularization and Bernstein methods the Abel fuzzy first kind integral equations will be solved. Note that the hybrid method increases accuracy of approximations.

\section{The Regularization Method}

The regularization methodwas established independently by Tikhonov in (Tikhonov, 1963) and Philips in (Philips, 1962) separately. In this method, integral equation of the first kind converts to integral equation of the second kind. By converting, the Fredholm first kind linear integral equation is defined as

$$
\left.f(x)=\int_{a}^{b} K(x, t) u(t) d t,\right)
$$

And non-linear integral equation has the form 


$$
f(x)=\int_{a}^{b} K(x, t) F(u(t)) d t,
$$

Converts to the approximation Fredholm integral equation

$$
\alpha u_{\alpha}(x)=f(x)-\int_{a}^{b} K(x, t) u_{\alpha}(t) d t,
$$

And

$$
\alpha u_{\alpha}(x)=f(x)-\int_{a}^{b} K(x, t) F\left(u_{\alpha}(t)\right) d t .
$$

Where, $\boldsymbol{\alpha}$ in (3) and (4) is the small real valued positive parameter which is called regularization parameter. It is clear that (3) and (4) are the second kind integral equations. They are presented as follows respectively

$$
\begin{array}{r}
u_{\alpha}(x)=\frac{1}{\alpha} f(x)-\frac{1}{\alpha} \int_{a}^{b} K(x, t) u_{\alpha}(t) d t \\
u_{\alpha}(x)=\frac{1}{\alpha} f(x)-\frac{1}{\alpha} \int_{a}^{b} K(x, t) F\left(u_{\alpha}(t)\right) d t
\end{array}
$$

Lemma: Fredholm first kind linear integral equation (1) substituted with Fredholm second kind linear integral equation (3) and $\boldsymbol{\alpha}$ is a small real valued positive parameter. Such that

$$
u(x)=\lim _{\alpha \rightarrow 0} u_{\alpha}(x)
$$

Proof: see (Wazwaz, 2011).

Tikhonov (Tikhonov, 1963) and Philips (Philips, 1962) proved that equations (5) or (6), when $\alpha \rightarrow 0$, is convergent into value of $u(x)$ and of (1), (2). Such that

$$
u(x)=\lim _{\alpha \rightarrow 0} u_{\alpha}(x)
$$

It is important to note that the fredholm integral equations off the first kind is an ill-posed problem. The solution for an ill-posed problem may not exist, and if it does exists; it may not be unique.

\section{Approximation by the Bernstein Polynomials and Integral Equations of the First Kind}

Assume function $f:[0,1] \rightarrow \mathbb{R}$. Bernstein polynomial $B_{n}(f)$ of the form:

$$
B_{n}(f(x))=\sum_{i=0}^{n} f\left(\frac{i}{n}\right) p_{n, i}(x),(7)
$$

It approximates function fon the interval of $[0,1]$ and $p_{n, i}$ is the polynomial with order $n$ presents as follows:

$$
p_{n, i}(x)=\left(\begin{array}{l}
n \\
i
\end{array}\right) x^{i}(1-x)^{n-1}, \quad i=0,1, \cdots, n .
$$

Bernstein applied this approximation to give a proof to Weirestrass theorem (Bernstein, 1912).

Following theorem proves that the sequence $\left\{\left.B_{n}(f)\right|_{n=1} ^{\infty}\right.$ converges to $f$ function.

Theorem 3.1 For all ffunctions on $C[0,1]$, the sequence $\left\{B_{n}(f) ; n=1,2,3, \cdots\right\}$ is convergent to $f$ in which $B_{n}(f)$ is determined by (7).

Proof: see (Powel, 1981). 
This theorem follows that, for any $f \in c[0,1]$ and for any $>0$, there exists $n$ such that the in equality $\left\|B_{n}(f)-f\right\|<\varepsilon$.

Holds.

In chapter 1 of (Revlin, 1969), it has been proved that $n$ satisfy

$$
n>\frac{S}{\delta^{2} \varepsilon}
$$

is being proved and $S=\|f\|$, becausefon $[0,1]$ is always constant, we have

$$
\forall \varepsilon>0 ; S, t \in[0,1],|t-S|<\delta \rightarrow-\frac{\varepsilon}{2}<f(t)-f(s)<\frac{\varepsilon}{2}
$$

For more details see (Revlin, 1969);

Assume that maximum $\|$.$\| is on [0,1]$ In this case, the error bound is shown as fallows

$$
\left|B_{n}(f(x))-f(x)\right| \leq \frac{1}{2 n} x(1-x)\left\|f^{\prime \prime}\right\|
$$

This bound has presented in chapter 10 of (Devore, 1993) that shows for $f \in C^{2}[0,1]$ the convergent rate is $\frac{1}{n}$. On the other handVoronovskaya asymptotic error (Voronovskaya, 1932) is given by

$$
\lim _{n \rightarrow \infty} n\left(B_{n}(f(x))-f(x)\right)=\frac{1}{2} x(1-x) f^{\prime \prime}(x),
$$

For $x \in[0,1], f^{\prime \prime}(x) \neq 0$ the convergent rate is exactly $\frac{1}{n}$. Now consider second kind integral equation

$$
f(x)=g(x)+\lambda \int_{0}^{x} K(x, t) f(t) d t, \quad 0 \leq x \leq 1,
$$

For numerical solution (12), we replace $f(t)$ with Bernstein polynomial approximate (7). So we get that

$$
\sum_{i=0}^{n} f\left(\frac{i}{n}\right)\left(\left(\begin{array}{l}
n \\
i
\end{array}\right)\left(x^{i}(1-x)^{n-i}-\lambda \int_{0}^{x} K(x, t) t^{i}(1-t)^{n-i} d t\right)\right)=g(x)
$$

To find the unknowns $f\left(\frac{i}{n}\right) i=0, \cdots, n$, the equation (13) converts to linear system of $A x=y$ by choosing $x_{j}, j=0,1, \cdots, n$. In this linear form

$$
\begin{aligned}
& A=\left[\left(\begin{array}{c}
n \\
i
\end{array}\right)\left(x_{j}^{i}\left(1-x_{j}\right)^{n-i}-\lambda \int_{0}^{x_{j}} K\left(x_{j}, t\right) t^{i}(1-t)^{n-i} d t\right)\right] \\
& i, j=0,1, \cdots, n \\
& X=\left[f\left(\frac{i}{n}\right)\right]^{t}, i=0,1, \cdots, n \quad, \quad y=\left[g\left(x_{j}\right)\right]^{t}, j=0,1, \cdots, n
\end{aligned}
$$

After having the system $A x=y \quad$ solved, the values of $f\left(\frac{i}{n}\right) i=0, \cdots, n$ are determined.

And by replacing these values in (7) the approximate solution of integral equation (12) will be achieved. Since, 
in regularization method, integral equation of the first kind converts to integral equation of the second kind, in system (12) integral equation of the second kind is presented. Now recall that, in this paper we tend to solve integral equation of the first kind. The error bound is given in Theorem (2).

Theorem 3.2 Consider the integral equation of the second kind (12). Assume that $k(x, t)$ is continuous on the square $[0,1]^{2}$ and the solution to equation for some $\alpha>2$ belongs to $\left(C^{\alpha} \cap L^{2}\right)([0,1])$. If $A$ is matrix (14) and only if A assumed invertible, then

$$
\sup _{x_{i} \in[0,1]}\left|f\left(x_{i}\right)-B_{n}\left(f_{n}\left(x_{i}\right)\right)\right| \leq \frac{1}{8 n}\left(M\left\|A^{-1}\right\| \cdot\left\|f^{\prime \prime}\right\|+\left\|f_{n}^{\prime \prime}\right\|\right),
$$

Where $x_{i}=\frac{i}{n} i=0, \cdots, n, \quad$ is exact solution of $M=\sup |\lambda K(x, t)|, \quad x, t \in[0,1]$ and $B_{n}\left(f_{n}(x)\right)$ are the solution of assumed method.

Proof: see (Maleknejad, Hashemizadeh \& Ezzati, 2011).

\section{Fuzzy Integral Equations of the First Kind}

In this section, properties of symmetrical fuzzy numbers are discussed.

Definition 4.1. (Klir, Clair \& Yuan, 1997) A fuzzy number is a mapping $u: \mathbb{R} \rightarrow I=[0,1]$ that demands following conditions:

I. $\mathrm{U}$ is semi- continuous

II. Outside some intervals such as $[c, d] \subset \mathbb{R}$ we have $u(x)=0$.

III. Real numbers ( $(\mathrm{a}, \mathrm{b})$ exist when $c \leq a \leq b \leq d$ if

1. $u(x)$ Is a monotonicallyincreasing on $[c, a]$;

2. $u(x)$ Is a monotonicallydecreasing on $[b, d]$;

3. $u(x)=1, a \leq x \leq b$.

Let $E^{l}$ denote the sets of all fuzzy numbers based on described definition (Klir, Clair \& Yuan, 1997). An alternative definition which yields the same $E^{l}$ is given by Kaleva (Kaleva, 1981; Ma, Friedman \& Kandel, 2003).

Definition 4.2 A desired fuzzy number in a parametric form of functions is stated as $(\underline{u}(r), \bar{u}(r)), 0 \leq r \leq 1$ and three following conditions are included:

(i) $\underline{u}(r)$ Is on a non-descending function, left continuous and bounded $[0,1]$.

(ii) $\quad \bar{u}(r)$ Is on a non-ascending function, left continuous and bounded $[0,1]$.

(iii) For all $0 \leq r \leq 1$ we have $\underline{u}(r) \leq \bar{u}(r)$. 
Remark 1. (Abbas, Babolian \& Alavi, 2007)Let $u(r)=(\underline{u}(r), \bar{u}(r)), 0 \leq r \leq 1$ be a fuzzy number, we take,

$$
u^{c}(r)=\frac{\underline{u}(r)+\bar{u}(r)}{2}, u^{d}(r)=\frac{\bar{u}(r)-\underline{u}(r)}{2}
$$

With the above definitions it is clear that $\bar{u}(r)=u^{c}(r)+u^{d}(r), \underline{u}(r)=u^{c}(r)-u^{d}(r)$ and $u^{d}(r) \geq 0$; and also a fuzzy number $u \in E^{l}$ is called symmetric if $u^{c}(r)$ for $0 \leq r \leq 1$ does not depend on $\mathrm{r}$.

Remark 2. For every desired fuzzy number, $u_{1}, V \in E^{l}$, we define below parameter for a interval between two fuzzy numbers $u$ and $V$ :

$$
D(u, V)=\sup \max \{|\bar{u}(r)-\bar{v}(r)|,|\underline{u}(r)-\underline{v}(r)|\} \quad 0 \leq \mathrm{r} \leq 1
$$

It is shown (Puri \& Ralescu, 1986) that $\left(E^{1}, D\right)$ is a complete metric space.

Abel integral equations are the most applied integral equations of the first kind (Wazwaz, 1997).

\section{Abel Fuzzy Integral Equations}

Let us define Abel integral

$$
f(x)=\int_{0}^{x} \frac{u(t)}{(x-t)^{\alpha}} d t ; \quad a \leq x \leq b
$$

Where $\alpha$ is a known constant and $O<\alpha<1 . f(x)$ Is a predetermined data function and $u(x)$ is the unknown function which should be calculated. The expression $(x-t)^{-\alpha}$ is calledthe kernel of Abel integral equation, or simply Abel kernel, that is singular as $t \rightarrow x$.

If $f(x)$ is acrisp function (non-fuzzy) then the solutions to equation (16) are crisp too. But if $f(x)$ is fuzzy function then the equation may have only fuzzy solutions.

Parametric form of Abel integral equation defines by

$$
\left(f \_(x, r), \bar{f}(x, r)\right)=\left(\int_{0}^{x} \frac{\underline{u}(t, r)}{(x-t)^{\alpha}} d t, \int_{0}^{x} \frac{\bar{u}(t, r)}{(x-t)^{\alpha}} d t\right),
$$

Where $0 \leq r \leq 1$ and $\alpha$ is known constants that $\tilde{f}(x)=\left(f_{-}(x, r), \bar{f}(x, r)\right) 0<\alpha<1$ is a predetermined data function; and $\tilde{u}(x)=(\underline{u}(x, r), \bar{u}(x, r))$ is the solutions to (17) which should be determined.

By assumption $\alpha=\frac{1}{2}$ in Eq. (17), the standard form of Abel non-linear fuzzy integral equation will be introduced as follows

$$
\left(f_{-}(x, r), \bar{f}(x, r)\right)=\left(\int_{0}^{x} \frac{F(\underline{u}(t, r))}{\sqrt{x-t}} d t, \int_{0}^{x} \frac{F(\bar{u}(t, r))}{\sqrt{x-t}} d t\right),
$$

Where the function $\left(f_{-}(x, r), \bar{f}(x, r)\right)$ is a function with real and known values and 
$(F(\underline{u}(x, r)), F(\bar{u}(x, r)))$ is the non-linear function of $(\underline{u}(x, r), \bar{u}(x, r))$.we recall that unknown function $(\underline{u}(x, r), \bar{u}(x, r))$ appears only in integral.

In order to solve the fuzzy non-linear integral equation of the first kind (18), first we need to convert it to the fuzzy non-linear integral equation of the second kind using regularization method, and then Bernstein approximations are employed to define the solution.

As it can be seen, fuzzy non-linear integral equation of the first kind(3) converts to the fuzzy non-linear integral equation of the second kind (6) and the solutions of $u_{\alpha}$ for system (6) when $\alpha \rightarrow 0$ is the exact solutions of (2).

Fuzzy form of the second kind using regularization method (18) is shown as

$$
\left(\left(\underline{u}_{\alpha}(t, r)\right),\left(\bar{u}_{\alpha}(t, r)\right)\right)=\frac{1}{\alpha}\left(f_{-}(x, r), \bar{f}(x, r)\right)-\frac{1}{\alpha} \int_{0}^{x} \frac{\left(F\left(\underline{u}_{\alpha}(t, r)\right), F\left(\bar{u}_{\alpha}(t, r)\right)\right)}{\sqrt{x-t}} d t
$$

Abel fuzzy integral equation (19) is solved by considering Bernstein polynomials then by decreasing value of $\alpha$, we expect the obtained approximations of $u_{\alpha}=\left(\underline{u}_{\alpha}, \bar{u}_{\alpha}\right)$ (19) tend to solutions of $u=(\underline{u}, \bar{u})$ (18). By having parametric form (19) and according to the systems (*) fuzzy Abel integral equation (19) transforms to the following non-fuzzy equations (Abbas, Babolian \& Alavi, 2007):

$$
\left(u_{\alpha}^{c}(t, r)\right)=\frac{1}{\alpha} f^{c}(x, r)-\frac{1}{\alpha} \int_{0}^{x} \frac{F\left(u_{\alpha}^{c}(t, r)\right)}{\sqrt{x-t}} d t,
$$

And

$$
\left(u_{\alpha}^{d}(t, r)\right)=\frac{1}{\alpha} f^{d}(x, r)-\frac{1}{\alpha} \int_{0}^{x} \frac{F\left(u_{\alpha}^{d}(t, r)\right)}{\sqrt{x-t}} d t .
$$

Equations(20) and (21) were solved by Bernstein polynomial approximations then we find $u_{\alpha}^{c}$ and $u_{\alpha}^{d}$.

We note that

$$
\underline{u}_{\alpha}(t, r)=u_{\alpha}^{c}(t, r)-u_{\alpha}^{d}(t, r) \quad, \quad \bar{u}_{\alpha}(t, r)=u_{\alpha}^{c}(t, r)+u_{\alpha}^{d}(t, r)
$$

Finally, after finding the value of $\underline{u}_{\alpha}$ and $\bar{u}_{\alpha}$, approximate solutions (18) are derived.

For small value of $\alpha$, we repeat above process and every time new $\underline{u}_{\alpha}$ and $\bar{u}_{\alpha}$ derived and every time $\alpha$ becomes smaller. As expected:

$$
\lim _{\alpha \rightarrow 0}\left(\underline{u}_{\alpha}, \bar{u}_{\alpha}\right)=(\underline{u}, \bar{u})
$$

Now, we present some examples of non-fuzzy and fuzzy integral equations so that we can study the efficiency of the hybrid method. As was noted in the article, fuzzy integral equations could be transformed to two non-fuzzy integral equations.

First, to show the effect of hybrid method in solving the integral equations of first kind, here we will illustrate some examples of solving non-fuzzy integral equations of the first kind using ybrid method; then we discuss hybridregularization method and Bernstein polynomials in solving non-fuzzy equations of the first kind and then the main examples that are fuzzy Abel integral equations will be solved using hybrid method.

Example 5.1 Consider the Fredholm integral equation of the first kind (Karimi \& Jozi 2015) 


$$
\int_{0}^{1} e^{t s} u(s) d s=\frac{e^{t+1}-1}{t+1}
$$

With the uniquesolution $u^{*}(t)=e^{t}$. If we transform equation (22) to the following second kind equation by regularization method

$$
u_{\alpha}(x)=\frac{1}{\alpha} \cdot \frac{e^{x+1}-1}{x+1}-\frac{1}{\alpha} \int_{0}^{1} e^{x s} u_{\alpha}(s) d s
$$

Then transforming second kind equation (23) to linear equations system, results in solving the second kind equation (23).

For more description, we divide interval $[0,1]$ to 10 parts. The unknowns of linear equations are going to be $f\left(x_{i}\right) i=0, \cdots, 10$ then after solving the equations the unknowns will be calculated.

In this section for approximating a definite integral, Simpson method was applied and Gaussian elimination method is employed to solve the linear equations.

By starting from $\alpha=1$ the linear equations derived from system (23) is being solved and then we need to decrease the value of $\alpha$ in order to achieve exact solution?

Convergence steps, diagrams of exact solutions and the diagram obtained from applying regularization method for $\alpha=1,0.1,0.001,0.000001$ are illustrated as follows:
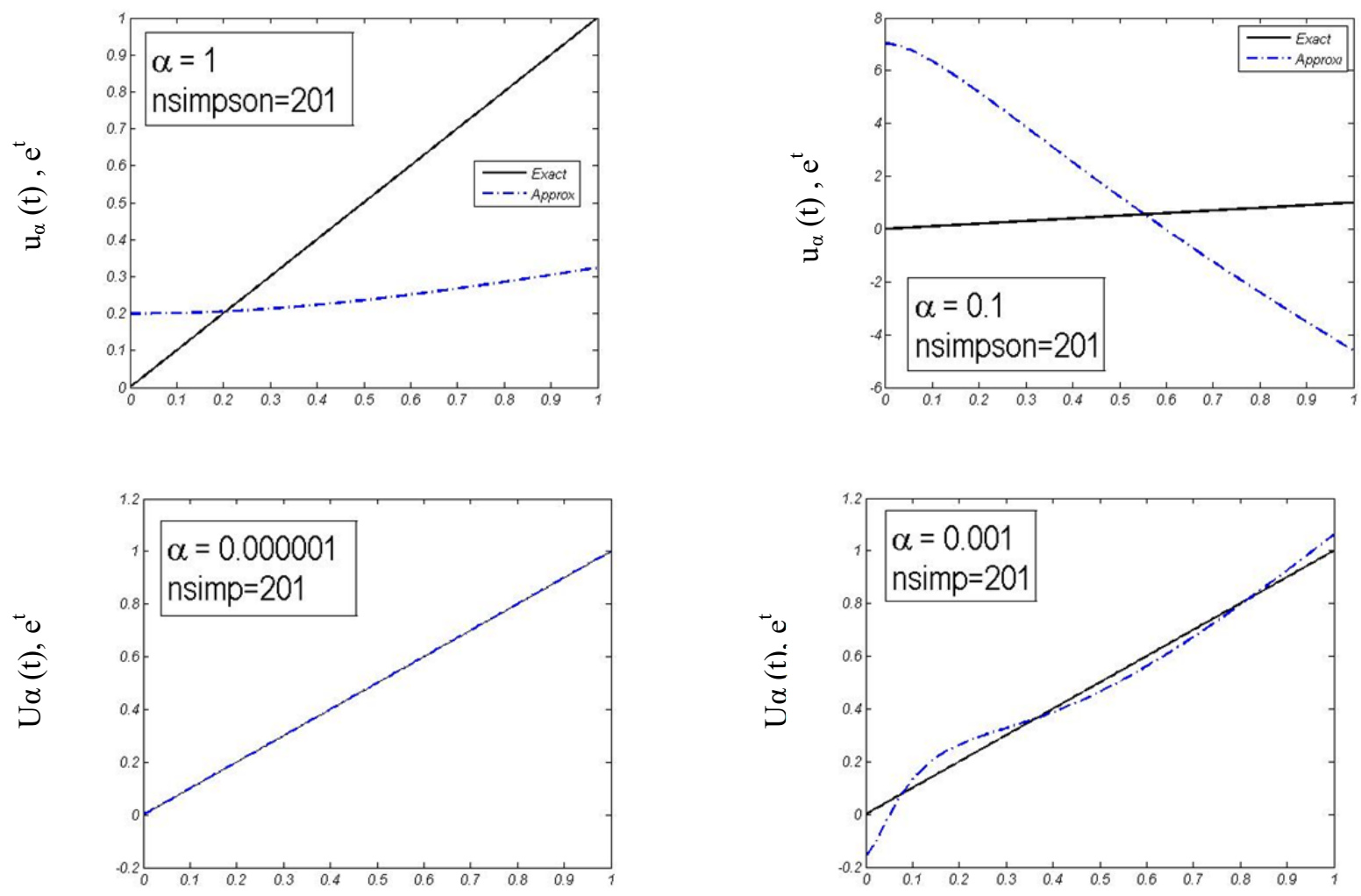

Figure 1. Plotting the convergence by two regularization methods for 
Due to this example solved by using least squares method in (Karimi \& Jozi 2015) so the results obtained from regularization method compared with system (Karimi \& Jozi 2015) in the following table.

Table 1. Thenumerical results obtained from regularization method compared with system (Karimi \& Jozi, 2015)

\begin{tabular}{llcc}
\hline $\mathrm{t}$ & $\left|u^{*}(t)-u_{3}(t)\right|$ & $\left|u^{*}(t)-u^{s}(t)\right|$ & $\begin{array}{c}\text { Regularization } \\
\text { method with } \\
\alpha=0.000001\end{array}$ \\
\hline 0 & $3.99 \times 10^{-4}$ & $1.06 \times 10^{-2}$ & $6.11 \times 10^{-4}$ \\
0.25 & $2.08 \times 10^{-4}$ & $2.46 \times 100^{0}$ & $1.78 \times 10^{-4}$ \\
0.5 & $2.39 \times 10^{-6}$ & $6.44 \times 10^{+1}$ & $5.99 \times 10^{-5}$ \\
0.75 & $3.82 \times 10^{-4}$ & $2.00 \times 10^{+1}$ & $6.72 \times 10^{-5}$ \\
1 & $7.49 \times 10^{-4}$ & $9.55 \times 10^{0}$ & $2.93 \times 10^{-4}$ \\
\hline
\end{tabular}

It can be seen that the results obtained from regularization method are better than two methods $u^{s}(t)$ and $u_{3}(t)$ in (Karimi \& Jozi, 2015).

Example 5.2 We consider the non-fuzzy integral equation of the first kind with (Maleknejad, Hashemizadeh \& Ezzati, 2011)

$$
\int_{0}^{x} e^{x-t} f(t) d t=\sin x \quad 0 \leq x \leq 1
$$

Where the exact solution is $f(x)=\cos x-\sin x 0 \leq x \leq 1$, if we use regularization method, the equation (26) transforms to following second kind equation:

$$
f_{\alpha}(x)=\frac{1}{\alpha} \sin x-\frac{1}{\alpha} \int_{0}^{x} e^{x-t} f_{\alpha}(t) d t
$$

Generally, in fuzzy form, by converting fuzzy equations to non-fuzzy equations, two equations such as (25) will be obtained. Therefore, equation of the second kind (25) would be solved using Bernstein polynomials. As previous examples, at first we solve the equation (25) for $\alpha=1$ and with a small value of $\alpha$, we find exact solution and achieve to the solution of (24) with an efficient accuracy.

Convergence steps of $f_{\alpha}$ and comparing it with exact values $f$ for $\alpha=1, \alpha=0.1$ and $\alpha=0.0001$ are presented in the following figures:
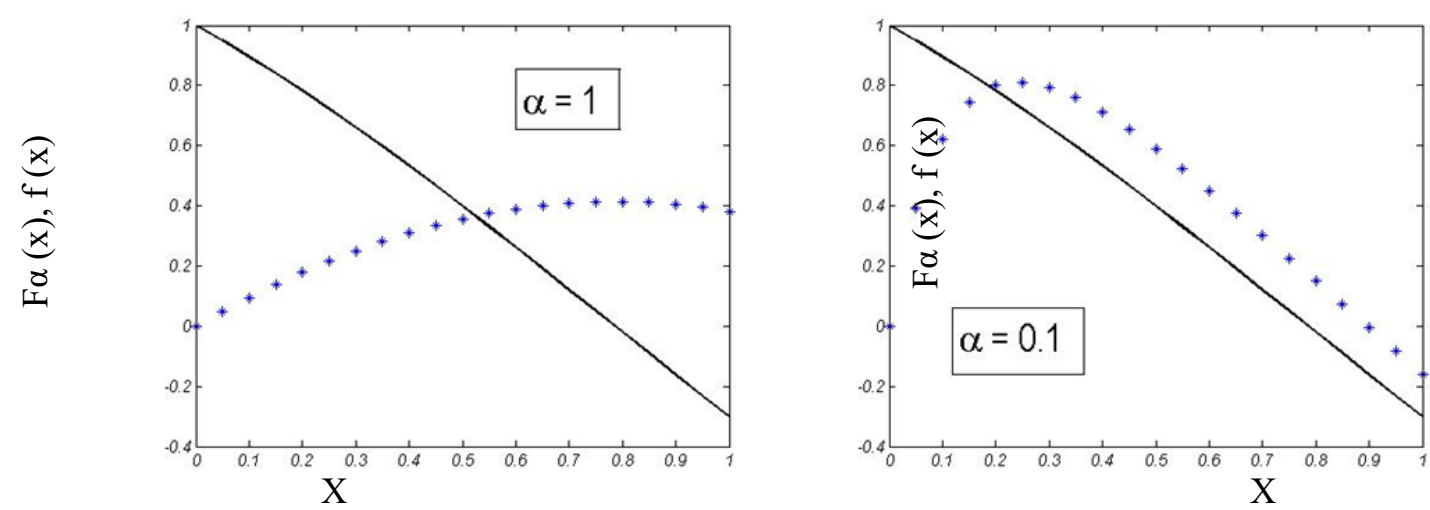


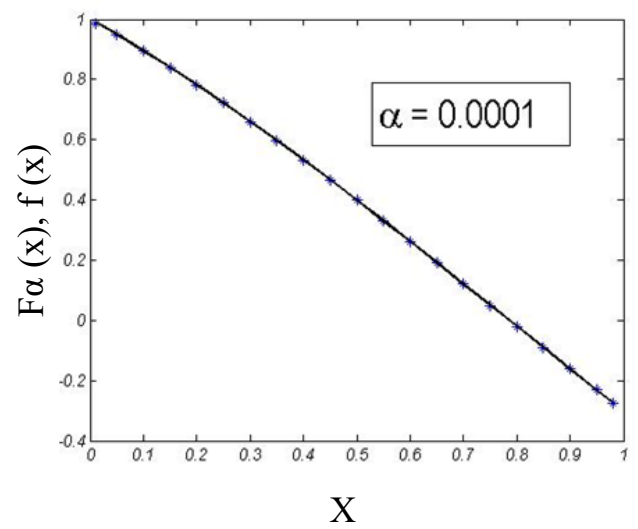

Figure 2. The error function graph for example 5.2 for $\alpha=1, \alpha=0.1$ and $\alpha=0.0001$

Finally by assumption of $\alpha=1 \times 10^{-13}$ on the interval $[0,1]$ the obtained results are:

Table 2. The results obtained from regularization method\& Bernstein for example

\begin{tabular}{|c|c|c|}
\hline$x_{i}$ & regularization & $\begin{array}{l}\text { method \& Bernstein polynomials } \\
\qquad \alpha=1 \times 10^{-13}\end{array}$ \\
\hline 0 & & $5.7057 \times 10^{-12}$ \\
\hline 0.1 & & $1.1879 \times 10^{-14}$ \\
\hline 0.2 & & $1.6953 \times 10^{-13}$ \\
\hline 0.3 & & $1.8807 \times 10^{-13}$ \\
\hline 0.4 & & $1.8129 \times 10^{-13}$ \\
\hline 0.5 & & $1.7630 \times 10^{-13}$ \\
\hline 0.6 & & $1.6636 \times 10^{-13}$ \\
\hline 0.7 & & $1.6327 \times 10^{-13}$ \\
\hline 0.8 & & $1.9531 \times 10^{-13}$ \\
\hline 0.9 & & $1.0222 \times 10^{-12}$ \\
\hline 0.98 & & $3.9913 \times 10^{-11}$ \\
\hline
\end{tabular}

This example in (Maleknejad, Hashemizadeh \& Ezzati, 2011) is solved by applying Bernstein polynomial approximation.

If $n=10$ the value of $\left\|e_{n}\right\|$ (total square error) is $8.09019 \times 10^{-7}$.

In above table, this error is $1.664326 \times 10^{-21}$.

The solution derived from hybrid of regularization and Bernstein polynomials approximations seem to be much more accurate than applying the solid Bernstein polynomials. So in this example the solution is better and more accurate; and also the implementation time is lasts about 2 minutes and makes cause no problem in calculating.

Now we consider the examples of Abel fuzzy integral equations. Operation of hybridal method will be compared with examples in (Jafarian \& Measoomy, 2014). Jafarian et al, in (Jafarian \& Measoomy, 2014) solved the Abel fuzzy integral equations by multilayer neural networks.

Example 5.3 consider the following fuzzy Abel integral equation (Jafarian \& Measoomy, 2014) 


$$
\left(\frac{4 r s^{\frac{3}{2}}}{3},-\frac{4(r-2) s^{\frac{3}{2}}}{3}\right)=\int_{0}^{s} \frac{(\underline{u}(t, r), \bar{u}(t, r))}{\sqrt{s-t}} d t, \quad 0<s \leq 1,0 \leq r \leq 1
$$

With the exact solution of Eq. (26) is: $u(s, r)=(r s,(2-r) s)$. After applying regularization method and transforming (26) to the second kind equation and according to $\alpha$ parameter, we solve the second kind equation that obtained from Bernstein polynomial approximation. With a small value of $\alpha$, more accurate solutions will be derived. The obtained solution for $\bar{u}$ and $\underline{u}$ according to $\alpha=2.5,1,0.01$ shown in below figures:
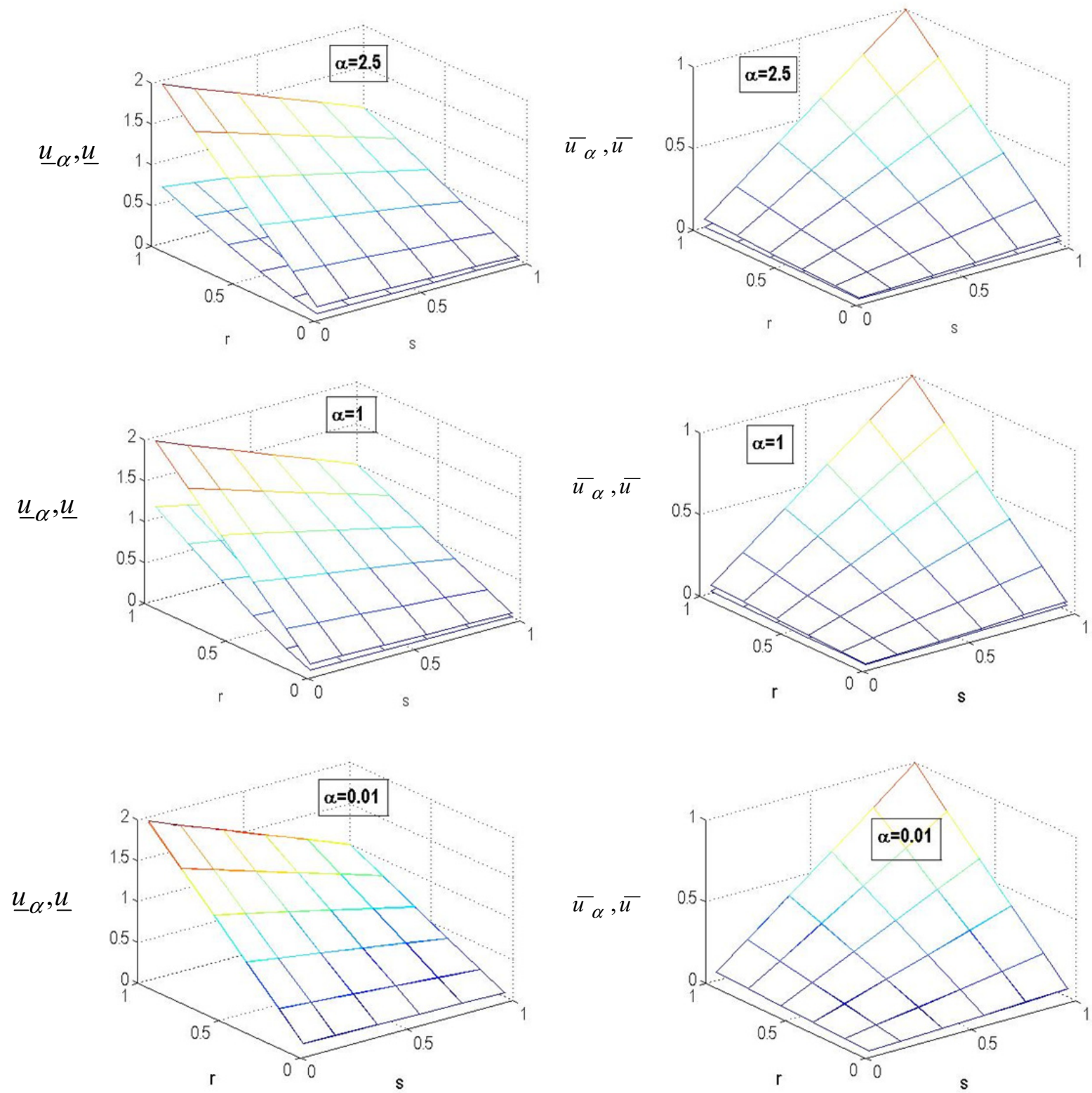

Figure 3. The exact and approximated solutions for example 5.3 for $\alpha=2.5,1,0.01$ 
Finally we achieve to the sufficient approximation of solutions ( $\bar{u}$ and $\underline{u}$ ) for $\alpha=1 \times 10^{-7}(26)$.

For comparing the results of hybrid method with Jafarian et al, method (Jafarian \& Measoomy, 2014) we illustrate the solutions in following table:

Table 3. The absolute error of numerical results for example 5.3

\begin{tabular}{ccccc}
\hline \multirow{2}{*}{$\left(s_{i}, r_{i}\right)=(0.1 i, 0.1 i)$} & \multicolumn{2}{c}{ FNN method (Jafarian \& Measoomy, } & \multicolumn{2}{c}{ hybridal method of This article } \\
\hline & $\underline{e}\left(s_{i}, r_{i}\right)$ & $\bar{e}\left(s_{i}, r_{i}\right)$ & $\underline{e}\left(s_{i}, r_{i}\right)$ & $\bar{e}\left(s_{i}, r_{i}\right)$ \\
& 0.00002 & 0.000057 & $1.551 \times 10^{-5}$ & $2.9448 \times 10^{-4}$ \\
$\mathrm{i}=1$ & 0.000043 & 0.000070 & $9.5748 \times 10^{-6}$ & $8.6173 \times 10^{-5}$ \\
$\mathrm{i}=2$ & 0.000070 & 0.000108 & $1.7151 \times 10^{-6}$ & $9.7193 \times 10^{-6}$ \\
$\mathrm{i}=3$ & 0.000100 & 0.000181 & $1.8559 \times 10^{-5}$ & $7.4237 \times 10^{-5}$ \\
$\mathrm{i}=4$ & 0.000251 & 0.000278 & $5.4882 \times 10^{-5}$ & $1.6464 \times 10^{-4}$ \\
$\mathrm{i}=5$ & 0.000343 & 0.000337 & $1.1116 \times 10^{-4}$ & $2.5938 \times 10^{-4}$ \\
$\mathrm{i}=6$ & 0.000535 & 0.000488 & $1.9034 \times 10^{-4}$ & $3.5350 \times 10^{-4}$ \\
$\mathrm{i}=7$ & 0.000854 & 0.000707 & $2.9567 \times 10^{-4}$ & $4.4350 \times 10^{-4}$ \\
$\mathrm{i}=8$ & 0.001411 & 0.002660 & $4.2899 \times 10^{-4}$ & $5.2432 \times 10^{-4}$ \\
$\mathrm{i}=9$ & 0.007282 & 0.009743 & $5.9745 \times 10^{-4}$ & $5.9745 \times 10^{-4}$ \\
$\mathrm{i}=10$ & & & & \\
\hline
\end{tabular}

Considering that in many points, the results from a hybrid method of regularization and Bernstein polynomials are better than the results of neural networks.

Example 5.4 Consider the following fuzzy Abel integral equation (Jafarian \& Measoomy, 2014)

$$
(\underline{f}(s, r), \bar{f}(s, r))=\int_{0}^{s} \frac{(\underline{u}(t, r), \bar{u}(t, r))}{\sqrt{s-t}} d t, \quad 0<s \leq 1,0 \leq r \leq 1,
$$

Where

$$
\begin{aligned}
& f_{-}(s, r)=\frac{1}{15}\left(2 \sqrt{s}\left(15 r-8 s^{2} \sqrt{\left(r^{2}+4 r+5\right)}+30\right)\right) ; \\
& \bar{f}(s, r)=-\frac{1}{15}\left(2 \sqrt{s}\left(15 r+8 s^{2} \sqrt{\left(r^{2}-8 r+17\right)}-60\right)\right) .
\end{aligned}
$$

For the given problem, the exact solution of equation (27)

$$
\begin{aligned}
& \underline{u}(s, r)=2+r-s^{2} \sqrt{5+4 r+r^{2}} ; \\
& \bar{u}(s, r)=4-r-s^{2} \sqrt{17-8 r+r^{2}} .
\end{aligned}
$$

Jafarian et al, also have solved this example by multilayer neural networks. After transforming the equation (27) to the second kind fuzzy integral equation, we use the hybrid method to solve it.

The convergence steps of ( $\bar{u}$ and $\underline{u}$ ) and their approximations for $\alpha=0.01,1,3$ shown in below figures: 

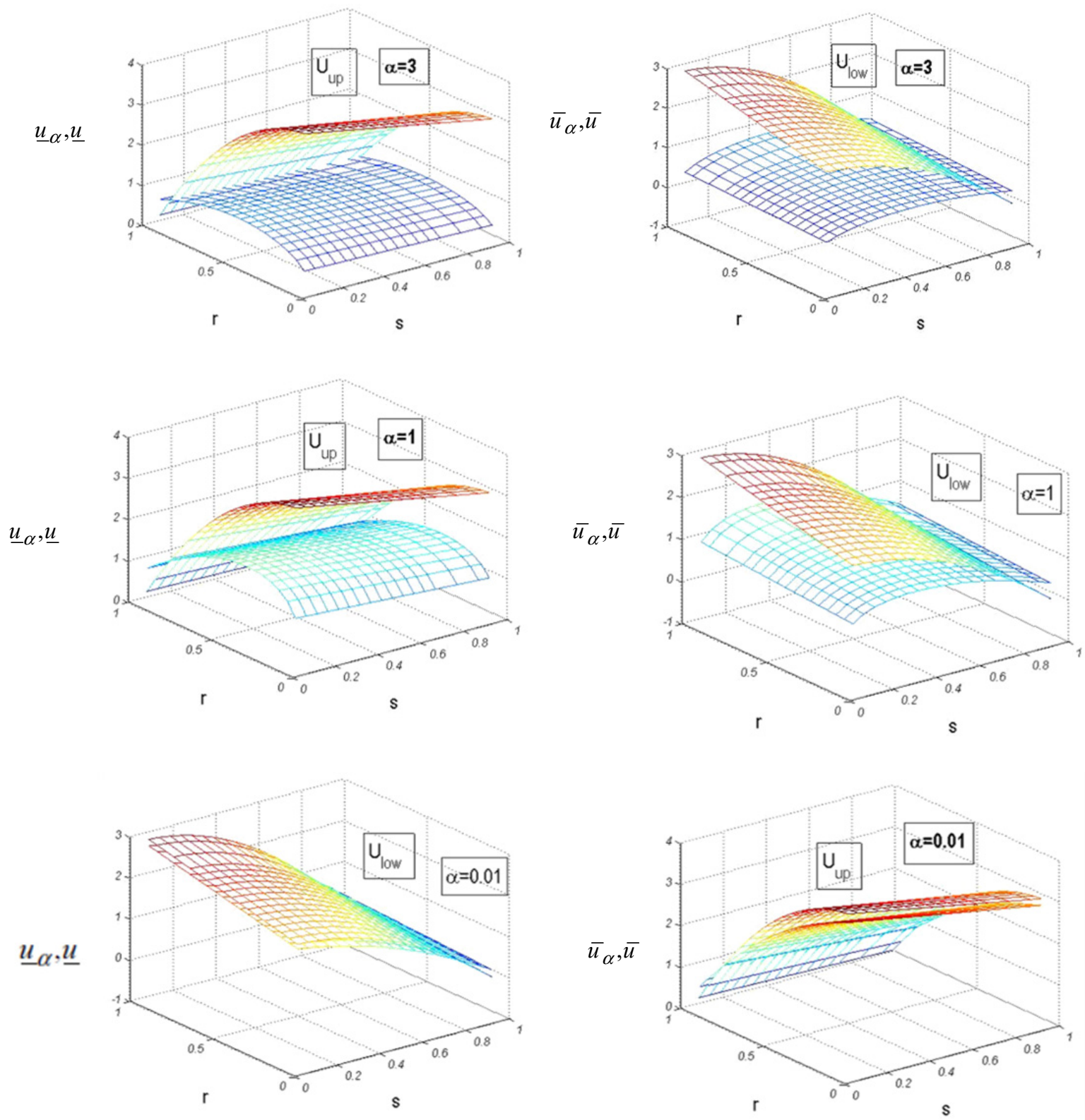

Figure 4. The exact and approximated solutions for example 5.4

By considering these figures we can say that in the regularization method and with a small value of $\alpha$ we are able to achieve to more accurate solution in first kind equation.

If the error is a little bit, we cannot study the figures because the errors less than 0.001 are not seen in the figures. So, for getting accurate comparing between the obtained results, consider the following table which includes the results derived from two methods. We assume $\alpha$ in hybrid method $\alpha=1 \times 10^{-8}$ : 
Table 5. The absolute error of numerical results for example 5.4

\begin{tabular}{ccccc}
\hline$\left(s_{i}, r_{i}\right)=(0.1 i, 0.1 i)$ & \multicolumn{2}{c}{ FNN method } & \multicolumn{2}{c}{$\begin{array}{c}\text { Regularization method \& Bernstein } \\
\text { polynomials }\end{array}$} \\
\hline & $\underline{e}\left(s_{i}, r_{i}\right)$ & $\bar{e}\left(s_{i}, r_{i}\right)$ & $\underline{e}\left(s_{i}, r_{i}\right)$ & $\bar{e}\left(s_{i}, r_{i}\right)$ \\
$\mathrm{i}=2$ & 0.000028 & 0.000031 & 0.003036 & 0.005246 \\
$\mathrm{i}=4$ & 0.000076 & 0.000089 & 0.000336 & 0.000501 \\
$\mathrm{i}=6$ & 0.000101 & 0.000173 & 0.000095 & 0.000111 \\
$\mathrm{i}=8$ & 0.000301 & 0.000405 & 0.000113 & 0.000114 \\
$\mathrm{i}=10$ & 0.000423 & 0.000702 & 0.001143 & 0.001143 \\
\hline
\end{tabular}

As it can be seen, in some points hybrid method is satisfied and in the other points FNN method is preferred. Of course we can say that the two methods in example 5 own the same efficiency.

\section{Conclusions and Recommendations}

In this paper the fuzzy integral equations of the first kind was solved by a hybrid method including regularization method and Bernstein polynomials approximation. While similar methods for solving integral equations of the first kind, especially Abel equations, have weak accuracy, so the obtained results for all examples of the first kind are acceptable and comparable to similar methods. In hybrid method the regularization results in converting the first kind integral equation to second kind integral equation and so we need a method for solving the second kind integral equation. We can choose this method over many other methods because the numbers of methods that solve the integral equations of the second kind are more than the numbers of methods to solve the first kind integral equations. Bernstein polynomials method is the acceptable method for solving the second kind equation and makes a solution for first kind equation that can be compared with other available methods.

The proposed hybrid method not only can solve the first kind fuzzy integral equations but also can solve the first kind non-fuzzy equations with a better accuracy. Especially this hybrid method cause acceptable solution to Abel fuzzy integral equations.

To change the hybrid method and study the ability of changing, we can replace the method of solving second kind equations. For instance, for approximating, we can use Chebyshev, hermit and Orthogonal polynomials instead of Bernstein polynomials.

The important advantage of this method over the other similar methods is the less run-time. For example in using the artificial neural networks the learning process increase the run- time but in the hybrid method run-time is not a problem at all.

\section{References}

Abbas, B. S., Babolian, E., \& Alavi, M. (2007). Numerical method for solving linear Fredholm fuzzy integral equations of the second kind. Chaos, Solitons and Fractal, 31(2007), 138-146.

Babolian, E., Sadeghi, Goghary, H., \& Abbasbandy, S. (2005). Numerical solution of linear Fredholm fuzzy integral equations of the second kind by Adomian method. Applied Mathematics and Computation, 161(2005), 733-744.

Bernstein, S. N. (1912). Demonstration du theoreme de weierstra. Fondee sur le calcul de probabilites. Commun Kharkov MathSo, (1912), 13, 1-2.

Devore, T. A., \& Lorentz, G. G. (1993). Constractive approximation. Berlin: Springer-Verlag.

Hanna, G., Roumeliotis, J., \& Kucera, A. (2005). Regularization an Fredholm integral equations of the first kind. Journal of Inequalities in pure and Applied Mathematics, 5, 131.

Jafarian, A., \& Measoomy, Nia, S. (2014). Artificial neural network approach to the fuzzy Abel integral equation problem. Journal of Intelligent and Fuzzy Systems, 27(2014), 83-91.

Jerri, A. (1999). Introduction to Integral Equations with Applications, INC, John Wiley and Sons.

Kaleva, O. (1981). Fuzzy differential equations. Fuzzy Sets Syst, 24, 301-317.

Karimi, S., \& Jozi, M. (2015). A new iterative method for solving linear Fredholm integral equations using the 
least squares method. Applied Mathematics and Computation, 250(2015), 744-758.

Klir, G. J., Clair, U. S., \& Yuan, B. (1997). Fuzzy set theory: foundations and applications, prentice-Hall Inc.

Maleknejad, K., Hashemizadeh, E., \& Ezzati, R. (2011). A New approach to the numerical solution of Volterra integral equation by using Bernstein approximation, Commun Nonlinear SCi Numer Simulat, 16(2011), 647-655.

Okecha, G. E. C. E. (2012). Onwukwe, on the solution of integral equations of the first kind with singular kernels of Cauchy-type. International Journal of Mathematics and Computer Science, 7(2), 129-140.

Philips, D. L. (1962). A technique for the numerical solution of certain integral equations of the first kind. J.A. comput. Mach, 9(1962), 84-96.

Polyanin, A. D., \& Manzhirov, A. V. (1998). Handbook of Integral Equations. CRC press, Bocaraton.

Powel, M. G. D. (1981). Approximation theory and methods. Cambridge university press.

Puri, M. L., \& Ralescu, D. (1983). Differential for Fuzzy Function, J.Math.Anal.Appl., 91, 552-558,.

Puri, M. L., \& Ralescu, D. (1986). Fuzzy random variables. JMO. Anal, Appl, 114, 409-22.

Revlin, T. J. (1969). An introduction to the approximation of functions. NewYork: Dover Publications.

Tikhonov, A. N. (1963). on the solution of ill-posed problem and the method of regularization, Soviet Math, 4(1963), 1035-1038.

Tikhonov, A. N. (1963). regularization of ill- posed problems, Soviet Math, Dokl, 4(1963), 1624-1627.

Voronovskaya, E. (1932). Determination de la forme asymptotion dapproximation des functions per les polynomes de M. Bernstein Doklady Akademii Nauk SSSR, 1932. p. 79-85.

Wazwaz, A. M. (1997). A First Couse in Integral Equation World Scientific publishing, Singapore.

Wazwaz, A. M. (2011). The Regularization - Homotopy Method for the Linear and Non-Linear Fredholm Integral Equations of the first kind, Vol 2011, Year 2011, Article ID can-00105, 11 pages.

\section{Copyrights}

Copyright for this article is retained by the author(s), with first publication rights granted to the journal.

This is an open-access article distributed under the terms and conditions of the Creative Commons Attribution license (http://creativecommons.org/licenses/by/4.0/). 